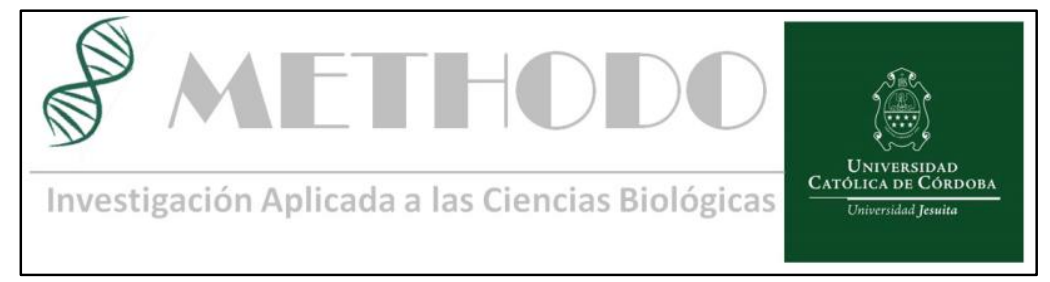

ARTICULO ORIGINAL Methodo 2018;3(2):32-34

DOI: 10.22529/me.2018.3(2) 03

Recibido 26 Sep.2017 | Aceptado10 Jun 2017 | Publicado 30 Jun 2018

\title{
Encefalitis inmunomediada, serie de casos
}

\section{Encephalitis immunemediated, a case series}

\author{
Vaudagna $\mathrm{MP}^{1-2}$, Vergara $\mathrm{G}^{1-2}$, Martínez $\mathrm{A}^{1-2}$, Paviolo $\mathrm{M}^{1-2}$, Miculan $\mathrm{J}^{1-2-3}$, Sfaello $\mathrm{Z}^{1}$, Sfaello $\mathrm{I}^{1-2}$.
}

\section{Introducción:}

La encefalitis es una inflamación grave que involucra el tejido cerebral que se desarrolla de manera aguda o subaguda generalmente en menos de 4-6 semanas, presentando un alto riego de morbi-mortalidad sin un tratamiento adecuado. Las etiologías pueden ser diversas, así como también los tratamientos requeridos. $\mathrm{La}$ identificación de la causa constituye entonces un desafío diagnóstico.

En la última década, ha habido un aumento en la identificación de formas de encefalitis asociadas con "anticuerpos de superficie neuronal". La patogénesis de las encefalitis autoinmunes radica en una respuesta inmune humoral, de anticuerpos dirigidos contra antígenos neuronales intracelulares, por un mecanismo etiopatogénico diferente, causado por una infección previa o asociado a tumores ocultos, como síndrome para neoplásico, este último más frecuente en adultos. ${ }^{1}$ La encefalitis por receptores de glutamato-Nmetil-D-aspartato es la forma más frecuente en niños, seguida a las encefalomielitis desmielinizantes agudas.

En la clínica de los pacientes predominan cambios súbitos de conducta y trastornos del movimiento, como así también convulsiones, lo que obliga a descartar causas infecciosas, traumáticas, junto con factores tóxicos, metabólicos y neoplásicos, que a veces conlleva a una demora diagnóstica. ${ }^{2-3}$ Los métodos complementarios diagnósticos pueden ser más o menos específicos para las diferentes fases de la enfermedad. La Resonancia Magnética de cerebro puede mostrar lesiones hipertensas en región límbica o temporal, o ser normal al inicio del cuadro clínico. En la punción lumbar se puede encontrar pleocitosis linfocítica leve a moderada, aumento de la concentración de proteínas o bandas oligoclonales en aproximadamente el $50 \%$ de los casos. A nivel electroencefalográfico, un patrón Delta Brush, puede estar presente en un $30-40 \%$ de los pacientes. ${ }^{4-5-6}$

En la bibliografía revisada, se recomienda iniciar la inmunoterapia empíricamente. Aunque algunos pacientes experimentan una recuperación completa espontánea, esto no es frecuente y no es posible identificar a los pacientes con pronóstico favorable.

La terapia de primera línea por lo general incluye esteroides, seguida o combinada, con administración de inmunoglobulina intravenosa. En caso de que el tratamiento de primera línea sea insatisfactorio, se debe iniciar tratamiento de segunda línea, con rituximab, ciclofosfamida o micofenolato, solo o en combinación. ${ }^{7}$

Con respecto al abordaje terapéutico, se deben esbozar estrategias adaptadas para cada paciente, considerando un enfoque menos agresivo para aquellos con un resultado generalmente mejor, teniendo en cuenta los posibles efectos secundarios versus beneficiosos de los medicamentos inmunoterápicos.

\section{Objetivos:}

Describir la evolución en pacientes pediátricos con diagnóstico de EI desde el año 2013 hasta el 2017.

\section{Materiales y métodos:}

Se realizó revisión retrospectiva, observacional de historias clínicas desde el año 2013 hasta el 2017. Se describieron 8 pacientes con EI, se revisó edad de los mismos, tiempo y método diagnóstico, clínica, tratamientos utilizados y evolución clínica. Las variables se presentaron en porcentajes.

Revista Methodo: Investigación Aplicada a las Ciencias Biológicas. Universidad Católica de Córdoba. Jacinto Ríos 571 Bo Gral. Paz. X5004FXS. Córdoba. Argentina. Tel.: (54) 3514517299 / Correo: methodo@ucc.edu.ar / Web: methodo.ucc.edu.ar | ARTICULO ORIGINAL Methodo 2018;3(2):32-34 


\section{Análisis estadístico:}

Se realizó estadística descriptiva.

\section{Resultados:}

Se describieron 8 pacientes con EA. Cuatro de sexo femenino $(50 \%)$ y cuatro masculino $(50 \%)$. La edad media del diagnóstico fue de 7.6 años con un rango de 2 a 14 años. El tiempo de seguimiento medio fue de 23.8 meses con un rango de 14 a 48 meses.

La demora en el diagnóstico varió entre 6 y 50 días, con un promedio de 26 días.

Siete presentaron EI definitiva, 6 EI Anti-NMDAr y uno EI de Hashimoto. El paciente restante, los títulos de anticuerpos en LCR fueron negativos y se define como EA posible, por la clínica.

Dentro de los 15 días de evolución, los pacientes presentaron convulsiones, predominando tónico clónicas generalizadas en 5 pacientes $(62 \%)$ asociadas a cambios conductuales y movimientos anormales, el más frecuente, disquinesias oro faciales en tres pacientes (38\%).

En todos los casos se realizó tratamiento de primera línea con cortico terapia y gammaglobulina EV. Cuatro, por falta de mejoría clínica requirieron plasmaféresis. En dos pacientes se realizó tratamiento de segunda línea con rituximab y en uno de ellos además ciclofosfamida.

La resonancia magnética anormal se informó en dos pacientes $(25 \%)$, predominando lesiones hiperintensas en FLAIR en regiones hipocámpicas. El patrón Deltha Brush se observó en un paciente $(12 \%)$.

En 6 de los 8 pacientes se observó remisión completa $(75 \%)$. Los pacientes que presentaron secuelas, el primer paciente presentó hemiplejía distónica-disquinética con mejoría en la respuesta a tratamiento de segunda línea y rehabilitación y el segundo paciente epilepsia refractaria.

Ninguno de los pacientes presentó asociación a tumores ocultos, ni episodios previos de Encefalitis.

\section{Conclusión:}

Desde su descripción a la fecha los reportes de EA han ido en aumento. Esta patología de presentación subaguda puede responder a distintas etiologías y presentarse también como un síndrome para neoplásico. El alto índice de sospecha puede orientar de manera adecuada los métodos complementarios y acelerar el proceso diagnóstico. La presencia de convulsiones asociadas a cambios cognitivos y conductuales debe elevar el índice de sospecha y orientar a su estudio específico. El inicio precoz del tratamiento se relaciona de manera directa con el pronóstico y evolución, ya que el $80 \%$ de los pacientes presentan una buena respuesta con recuperación total o casi total de los síntomas.

\section{Introduction:}

Since the first description, immune-mediated encephalitis (EI), among them, against the anti-NMethyl-D-Aspartate (NMDAr) receptor has been increasingly recognized, being considered the most frequent immunomediated inflammatory process after Encephalomyelitis acute disseminated.

\section{Objective:}

To describe trends in pediatric patients diagnosed with el.

\section{Materials and methods:}

Retrospective, observational clinical histories review was performed in the last 4 years. Eight patients with EI described. The mean age at diagnosis was 7.6 years. The delay in the diagnostic ranged from 6 to 65 days, with 26 days' average. Seven presented final EI, EI AntiNMDA el 6 and one Hashimoto el.

\section{Results:}

Within 15 days of evolution, patients had seizures associated with behavioral changes and abnormal movements, the most frequent orofacial dyskinesias in 38\%. In all cases first - line treatment was performed. Four required smafé pla resist. In three patients with second line treatment Rituximab was performed and one cyclophosphamide. The Deltha Brush Pattern was observed in a patient.

In 6 of the 8 patient's complete remission $(75 \%)$ was observed. There was no patient in association with occult tumors. Time tracking in an average of 22.8 months with a range of 4-48 months.

\section{Conclusions:}

Since its description to date, EI reports have been increasing. The presence of seizures associated with cognitive and behavioral changes should raise the index of suspicion and their specific study guide. Early initiation of treatment is associated directly with prognosis and evolution. 


\section{Bibliografía:}

1. Giorgia Olivieri, Ilaria Contaldo, y cools; "Autoimmune encephalopathies in children: diagnostic clues and therapeutic challenges"; Neuroimmunol Neuroinflammation, 2016;3:14755; DOI: 10.20517/2347-8659.2016.09

2. Yael Hacohen; "Paediatric autoimmune encephalopathies: clinical features, laboratory investigations and outcomes in patients with or without antibodies to known central nervous system autoantigens"; J Neurol Neurosurg Psychiatry 2013; 84:748-755. doi:10.1136/jnnp2012-303807

3. Jehan Suleiman, Russell C Dale y cools; "The recognition and treatment of autoimmune epilepsy in children"; Developmental Medicine \& Child Neurology 2015, 57: 431-440; DOI: 10.1111/dmcn. 12647

4.Jehan Suleiman; "Autoimmune epilepsy in children: Case series and proposed guidelines for identification"; Epilepsia, 54(6):1036-1045, 2013; doi: 10.1111/epi.12142

5. Stephen VanHaerents, Alexandra Stillman, y cools; "Early and persistent 'extreme delta brush' in a patient with anti-NMDA receptor encephalitis"; Epilepsy \& Behavior Case Reports Volume 2, 2014, Pages 67-70; https://doi.org/10.1016/j.ebcr.2014.01.002

6. Sarah E. Schmitt, Kimberly Pargeon, y cools; "Extreme delta brush. A unique EEG pattern in adults with anti-NMDA receptor encephalitis"; Neurology 79 September 11, 2012; DOI

7. Sarosh R. Irani y cools; "N-Methyl-D-aspartate antibody encephalitis: temporal progression of clinical and paraclinical observations in a predominantly non-paraneoplastic disorder of both sexes"; Brain 2010: 133; 1655-1667; doi:10.1093/brain/awq113-406

\section{Palabras claves:}

Encefalitis inmunomediada, Epilepsia, movimientos anormales, Cambios conductuales.

\section{Keywords:}

Immune - mediated Encephalitis, Epilepsy, Abnormal Movements, Behavioral Changes.
1 Unidad de cefalea - Liège - Bélgica

2 Servicio de Neurología, Clínica Universitaria Reina Fabiola, Universidad Católica de Córdoba, Argentina.

Correspondencia: Emiliano Ruiz Romagnoli. Clínica Universitaria Reina Fabiola. Oncativo 1248 -X5004FHP- Córdoba, Argentina. e-mail: emilianoruizomagnoli@gmail.com

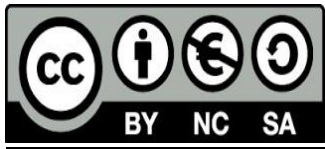

